\title{
A case of infra-nodal Wenckebach conduction block with alternating bundle branch block
}

Pichmanil Khmao ${ }^{1}$ (D), Chun Hwang ${ }^{2}$ and Hui-Nam Pak ${ }^{1 *}$ (1)

\begin{abstract}
Background: Atrioventricular (AV) node normally has decremental conduction property and a longer refractory period than His-Purkinje system (HPS). This results in AV conduction delay or block at the level of AV node in response to short-coupled atrial premature beats. Prolonged refractoriness in HPS can produce unusual physiological patterns of AV conduction such as conduction delay or infra-nodal block in the distal elements of HPS.
\end{abstract}

Case presentation: We present a case in which atrial premature stimulation produces infra-nodal Wenckebach conduction block which initiates long-short cycle sequence within the bundle branches resulted in alternating bundle branch block and atypical pattern of Ashman phenomenon.

Conclusions: This case highlights the importance of recognizing the unusual physiological AV conduction patterns of HPS. The long-short cycle sequence in the bundle branches of distal HPS and linking phenomenon can result in alternating bundle branch block without the presence of HPS disease.

Keywords: Ashman phenomenon, Atrioventricular node, Bundle branch block, His-Purkinje system, Infra-nodal block

\section{Introduction}

Atrioventricular conduction patterns and refractoriness within His-Purkinje system (HPS) in human were initially reported in the early 1970 s $[1,2]$. Normally, atrioventricular $(\mathrm{AV})$ node has a longer refractory period than HPS and prolonged refractoriness in HPS can produce unusual patterns of AV conduction. We present a case in which atrial premature stimulation produced infra-nodal Wenckebach conduction block which initiates long-short cycle sequence within the bundle branches resulted in alternating bundle branch block and atypical pattern of Ashman phenomenon.

*Correspondence: hnpak@yuhs.ac

1 Yonsei University Health System, 50-1 Yonsei-ro, Seodaemun-gu, 03722 Seoul, Republic of Korea

Full list of author information is available at the end of the article

\section{Case}

The patient is a 28-year-old female, who experienced recurrent palpitations with narrow QRS tachycardia, was referred for electrophysiologic study. At baseline, the sinus cycle length (CL), AH, and HV interval were $695 \mathrm{~ms}, 45$ $\mathrm{ms}$, and $50 \mathrm{~ms}$, respectively. Concentric retrograde atrial activation with decremental property was noted during programmed ventricular stimulation. By straight atrial pacing of CL $280 \mathrm{~ms}$, infra-nodal Wenckebach conduction block pattern was noted and followed by alternating left bundle branch block (LBBB) and right bundle branch block (RBBB) QRS morphology with a resumption of 1:1 AV conduction (Fig. 1a). Single atrial extra-stimulus (AES) progressively prolonged both $\mathrm{AH}$ and $\mathrm{HV}$ intervals, and $\mathrm{LBBB}$ pattern was observed at S2 $240 \mathrm{~ms}$ (Fig. 2a and b). Decrement of $10 \mathrm{~ms}$ of AES to $230 \mathrm{~ms}$ demonstrated AH jump with a shortening HV interval (Fig. 2c). Atrial effective refractory period (ERP) was reached at 500/200 ms before that of the AV conduction system. At the drive train of S1 


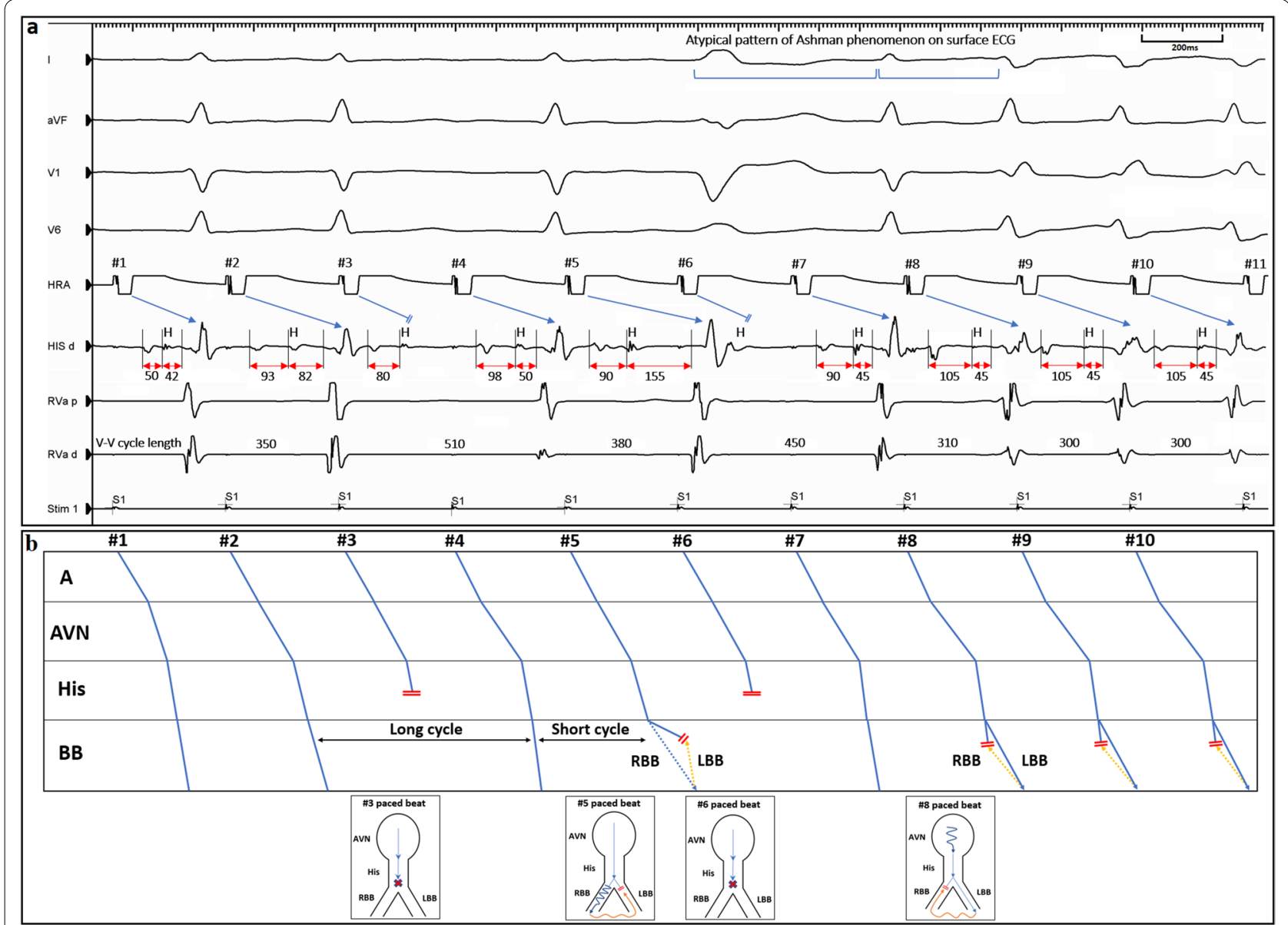

Fig. 1 a. Rapid atrial pacing at 280 ms produced infra-nodal Wenckebach conduction block followed by an alternating bundle branch block with a resumption of 1:1 AV conduction. b. Illustration of laddergram and diagrammatic representation of linking phenomenon and gap phenomenon of panel $\mathbf{a}$. Noted the long-short cycle sequence in the bundle branches produces an alternating bundle branch. The duration intervals are in milliseconds. A indicates atrium; AVN, AV node; BB, bundle branches; LBB, left bundle branch; RBB, right bundle branch; $V$, ventricles. See detailed discussion in the text

600 ms with double atrial extra-stimuli at S2S3 300/290 ms showed HV block (Fig. 2d).

Tachycardia was not inducible despite programmed stimulation protocol on the baseline state and under infusion of Isoproterenol. Catheter ablation was performed due to documented paroxysmal supraventricular tachycardia (PSVT) with dual AV nodal physiology and nodal echo beats. It is noteworthy that programmed electrical stimulation reproducibly demonstrated the same pattern of alternating bundle branch blocks and infra-nodal block as above mentioned after successful modification of AV nodal slow pathway.

\section{Discussion}

Wit et al. classified 3 types of AV conduction patterns in human [2]. In type 1 response, conduction delay and block were limited in the AV node while type 2 response was characterized by progressive conduction delay in both the AV node and specialized conduction system with block occurring in several instances in the latter. Type 3 response, the least common pattern, was characterized by a progressive delay in the AV node and a sudden marked delay in HPS. The manifestation of type 3 response is linked to a longer refractory period of HPS comparing to the AV node and/or relatively rapid conduction over the AV node. In this case, atrial premature beats with short coupling interval conducted through the AV node and encounters relative refractory period (RRP) of right bundle branch (RBB) while found ERP of left bundle branch (LBB) resulted in pronounced HV prolongation with $\mathrm{LBBB}$ pattern (Fig. 2b upper right panel). An alternative potential explanation might be LBB was blocked by the concealed retrograde activation from RBB (Fig. 2b lower right panel). The abrupt increase in $\mathrm{AH}$ 


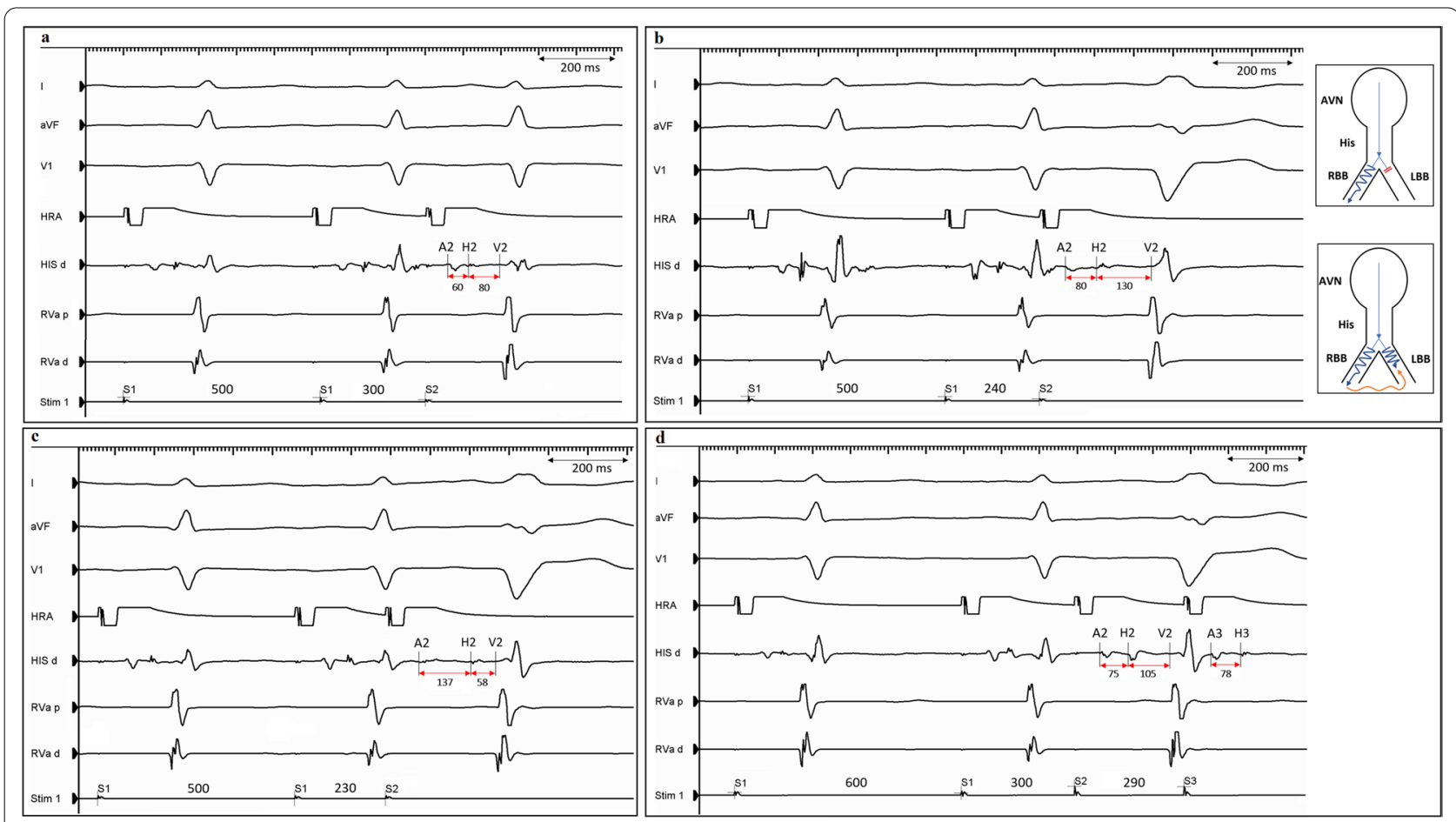

Fig. 2 a. Atrial programmed stimulation S1S2 500/300 ms showed HV interval prolongation (H2V2 80 ms) with conducted narrow QRS complex. b. Left panel: AES S1S2 500/240 ms showed pronounced HV interval prolongation (H2V2 130 ms) with LBBB pattern. Right panel: Diagrammatic representation of 2 possible mechanisms in pronounced $\mathrm{HV}$ interval prolongation with LBB aberrancy in response to atrial premature impulses. c. Decrement of 10 ms of S1S2 500/230 ms resulted in sudden increasing AH interval (A2H2 137 ms) with shortening HV interval (H2V2 58 ms). d. Atrial programmed stimulation S1S2S3 600/300/290 ms demonstrated a conduction with infra-nodal block. The duration intervals are in milliseconds. AES indicates atrial extra-stimulus

interval shortened HV interval due to important delay of conduction in the AV node allowed for recovery from the refractoriness in distal elements in the HPS (Fig. 2c). Although the HV interval was shortened, LBB aberrancy was remained suggesting the mechanism of asynchronous recovery from refractoriness in HPS [3].

An alternating pattern of bundle branch block straddling a normal QRS complex with a resumption of 1:1 AV conduction after infra-nodal Wenckebach block can be explained by functional bundle branch block due to a long-short cycle sequence of the HPS and gap phenomenon (Fig. 1b). In 1947, Gouaux and Ashman described the aberrancy of conduction usually follows a long-short ventricular cycle sequence [4]. In Fig. 1, the 3rd paced beat was blocked at infra-nodal level proximal to the bundle branches and produced a long cycle sequence in the bundle branches for the next beat. Following a preceding long cycle, the 5 th paced beat was conducted with pronounced prolongation of $\mathrm{HV}$ interval with $\mathrm{LBBB}$ because the impulse encountered RRP of RBB while was blocked at the proximal LBB which warranted concealed trans-septal retrograde activation from the RBB. This concealed retrograde invasion the so-called linking phenomenon shortens refractoriness in the LBB for the subsequent beat [5]. As a result of the linking phenomenon, the 8th paced beat was able to conduct normally through the LBB while the block occurred in the RBB which remained in the refractory period resulted in $\mathrm{RBB}$ aberrancy with normalization of HV interval. Note that from the 8th atrial paced beat showed decremental conduction through the AV node which facilitated the conduction through the distal His bundle. This kind of gap phenomenon produced a resumption of $1: 1 \mathrm{AV}$ conduction (Fig. 1b). The maintenance of RBB aberrancy in the following beats can be repetitive concealment by transseptal retrograde activation from the LBB. Furthermore, there was no typical pattern of long-short R-R sequence on the surface ECG in the alternating bundle branch block because the short cycle sequence produced a pronounced HV interval prolongation.

\section{Conclusion}

The present case highlights the importance of recognizing the unusual physiological AV conduction patterns of HPS. The long-short cycle sequence in the bundle 
branches of distal HPS and linking phenomenon can result in alternating bundle branch block without the presence of HPS disease.

\section{Abbreviations}

AES: Atrial extra-stimulus; AH: Atrio-His; AV: Atrioventricular; CL: Cycle length; ERP: Effective refractory period; HPS: His-Purkinje system; HV: His-Ventricular; LBB: Left bundle branch; LBBB: Left bundle branch block; PSVT: Paroxysmal supraventricular tachycardia; RBB: Right bundle branch; RBBB: Right bundle branch block; RRP: Relative refractory period.

\section{Acknowledgements}

Not applicable.

\section{Authors' contributions}

Pichmanil Khmao was a major contributor writing the manuscript. Chun Hwang and Hui-Nam Pak reviewed and revised the manuscript. All authors read and approved the final manuscript.

\section{Funding}

Not applicable.

\section{Declarations}

Ethical approval and Consent to participate

Written informed consent before procedure was given.

\section{Competing interests}

Authors declare no conflict of interests for this article.

\section{Author details}

${ }^{1}$ Yonsei University Health System, 50-1 Yonsei-ro, Seodaemun-gu, 03722 Seoul, Republic of Korea. ${ }^{2}$ Department of Cardiology, Revere Health, Provo, UT, USA.

Received: 8 April 2021 Accepted: 30 April 2021

Published online: 17 May 2021

\section{References}

1. Damato AN, Varghese PJ, Caracta AR, Akhtar M, Lau SH. Functional 2:1 A-V block within the His-Purkinje system. Simulation of type II second-degree A-V block. Circulation. 1973:47:534-42.

2. Wit AL, Weiss MB, Berkowitz WD, Rosen KM, Steiner C, Damato AN. Patterns of atrioventricular conduction in the human heart. Circ Res. 1970;27:345-59.

3. Stark S, Farshidi A. Mechanism of alternating bundle branch aberrancy with atrial bigeminy: electrocardiographic-electrophysiologic correlates. J Am Coll Cardiol. 1985:5:1491-5.

4. Gouaux JL, Ashman R. Auricular fibrillation with aberration simulating ventricular paroxysmal tachycardia. Am Heart J. 1947:34:366-73.

5. Rosenbaum MB, Elizari MV, Lazzari JO, Nau GJ, Halpern MS, Levi RJ. The differential electrocardiographic manifestations of hemiblocks, bilateral bundle branch block, and trifascicular blocks. New York: Grune \& Stratton; 1972.

\section{Consent for publication}

All data generated are anonymized.

Ready to submit your research? Choose BMC and benefit from:

- fast, convenient online submission

- thorough peer review by experienced researchers in your field

- rapid publication on acceptance

- support for research data, including large and complex data types

- gold Open Access which fosters wider collaboration and increased citations

- maximum visibility for your research: over $100 \mathrm{M}$ website views per year

At BMC, research is always in progress.

Learn more biomedcentral.com/submissions 